\title{
Schluss und Ausblick: komplexe Passungsverhältnisse
}

\subsection{Zusammenfassung der Ergebnisse}

Ziel der vorliegenden Arbeit war es, die Erkenntnisse von Geschlechter- und Bildungsforschung zu Studien(fach)wahlen miteinander zu verknüpfen und in der Analyse die Dimensionen von sozialer Herkunft und Geschlecht systematisch zu verbinden. Studien(fach)wahlen wurden so als Ausdruck eines spezifischen Passungsverhältnisses von vergeschlechtlichten und vergeschlechtlichenden Milieuhabitus und Fachkulturen bzw. beruflichen Positionen begriffen und auf dieser Grundlage wurden die damit verbundenen Muster der Studien(fach)wahl in unterschiedlichen sozialen Milieus untersucht. So sollte die Frage beantwortet werden, wie die Wahl eines Studiums und Studienfachs vor dem Hintergrund von sozialem Milieu und Geschlecht zu verstehen und zu erklären ist.

\section{Habitusspezifische Einbettung von Studien(fach)wahlen}

Wie der dargestellte Forschungsstand von Geschlechter- und Bildungsforschung deutlich macht, wird der Weg in das Studium und ein Studienfach von unterschiedlichen Faktoren beeinflusst - den Noten und Erfahrungen in der Schule, den fachlichen Interessen, von Orientierungspersonen, dem sozialen Umfeld inklusive der Peers usw. Aus der Perspektive der Habitus- und Milieuforschung sind all diese Faktoren eingebettet in die Habitualisierung an einem spezifischen sozialen Ort bzw. in einem bestimmten sozialen Milieu.

Ganz grundsätzlich ergeben die Analysen der Fälle, dass der Weg in ein Studium maßgeblich geprägt ist vom sozialen Milieu und dass ,Frauen“ und ,Männer' des gleichen Milieus bzw. des gleichen Spektrums sozialer Milieus ${ }^{1}$ in

\footnotetext{
${ }^{1}$ Wie auch in der Analyse kommt es bei dieser Schlussfolgerung nicht auf die konkrete Zuordnung eines Falls zu einem spezifischen sozialen Milieu an, sondern vielmehr auf 
ihren Studien(fach)wahlen mehr verbindet, als sie jeweils trennt. Grundlegende habitusspezifische Muster und die spezifische Kombination von Habituszügen wie die von Gemeinschaftsorientierung, Disziplin, Aufstiegsstreben oder Traditionsorientierung sind Ergebnis der Sozialisation an einem bestimmten sozialen Ort und prägen die Studienaufnahme für die Angehörigen beider Genusgruppen entscheidend. Innerhalb dieses milieuspezifischen Möglichkeitsraums finden vergeschlechtlichte fachliche Prägungen, Ablenkungen, Unterstützungen und Benachteiligungen statt - etwa durch Vorbilder, fachkulturelle Sozialisationsprozesse usw. Quer dazu wiederum liegt die heteronormativ-vergeschlechtlichte Arbeitsteilung von Erwerbs- und Familienarbeit, die milieuübergreifend zu finden, aber milieuspezifisch gefärbt ist: Sie beeinflusst entscheidend die Ungleichverteilung von sozialen Positionen innerhalb jedes Milieus und ist Teil des Gesamtbildes, das die Passung zu einer Fachkultur und einem Beruf ausmacht.

Milieuspezifische Unterschiede zwischen den Fällen finden sich etwa im familiären Umgang mit der Schulbildung: Dieser findet in verschiedener Form statt, nämlich etwa als zielgerichtete Einflussnahme auf die Schullaufbahn des Kindes, um den höchsten Schulabschluss zu erreichen (gefunden im Muster von „Aufstiegsstreben und Disziplin“), als offene und kooperative Unterstïtzungspraktiken jenseits eines bestimmten Abschlusses (gefunden im Muster „Gemeinschaft und Ordnung"), als selbstverständliche Sozialisation hin zum Studium mit entsprechender familiärer Unterstützung (gefunden im Muster „Traditions- und Stabilitätsorientierung") oder als begrenzte Unterstützung mit Stolz oder partieller Skepsis gegenüber einem höheren Schulabschluss (gefunden beim Muster „Autonomie durch Leistung“) ${ }^{2}$

Auch vergeschlechtlichte Orientierungspersonen und fachliche Prägungen beeinflussen die Studien(fach)wahl nicht unabhängig von der milieuspezifischen Einbettung: Dass Studienfachwahlen ,nicht das Resultat einer einfachen Reproduktion herkunftsfamiliärer fachkultureller Traditionen und Berufsleitbilder [darstellen], sondern sich aus individuell hochkomplexen, vielschichtigen und sehr facettenreichen familialen Beziehungs- und Interaktionssystemen [speisen]“ (Puchert 2017: 364), spiegelt sich in allen Fällen wieder. Eine offene Anlehnung an familiäre Fachtraditionen zeichnet sich insbesondere in der ständischkleinbürgerlichen Linie ab, während sonst in unterschiedlicher Intensität eher

seine relationale Verortung im Verhältnis zu den anderen Fällen und auf seine ungefähre Position im sozialen Raum, die durchaus mit Unschärfen einhergeht.

${ }^{2}$ Beim Muster „Unkonventionalität und Idealismus“ sind es die bereits dargestellten unterschiedlichen elterlichen (und milieuspezifischen) Einflüsse im Fall Tabea, die eine gemeinsame Benennung des familiären Umgangs mit Schulbildung nicht zulassen. 
implizite Prozesse der Orientierung an familiären fachlichen Prägungen festzustellen sind, bei denen teilweise Tätigkeiten reproduziert werden oder - im Zusammenspiel mit entsprechenden biografischen Erfahrungen, dem Einfluss von Peers etc. - relativ neue Wege eingeschlagen werden, etwa vor dem Hintergrund von Autonomiestreben oder der Verlagerung der familiären Strategien zur Statusabsicherung.

Und ebenso greifen institutionelle Lenkungen abhängig vom sozialen Ort der Befragten: Ob und mit welchen Umwegen etwa ein Studium aufgenommen wird, wird, wie so vieles, von institutionellen Faktoren mitbestimmt und herkunftsbedingte Unterschiede können so verstärkt werden. Das spiegelt sich etwa im Vergleich der Muster „Traditions- und Stabilitätsorientierung“ und „Autonomie durch Leistung" und den dortigen Wegen in das Bauingenieurwesen wider: Beim ersten ist der Besuch eines Gymnasiums mit entsprechendem Konzept mit einem Praktikum in einem akademischen Beruf verknüpft, während beim zweiten der Besuch einer Gesamtschule fast unweigerlich zu einem Praktikum in einem Ausbildungsberuf führt - beides bildet jeweils einen Baustein in der Orientierung hin zu den entsprechenden Qualifizierungen und Berufen. Im Zusammenspiel mit anderen Faktoren (wie Orientierungspersonen) kommt es im Fall der autonomieorientierten Arbeitertochter so zu einer Passung zum Bauingenieurwesen und im Fall der traditionsorientierten Akademikertochter zu einer höheren Passung.

\section{Habitusspezifische Nähe zur Sozialen Arbeit bzw. zum Bauingenieurwesen}

Vor diesem Hintergrund der milieuspezifischen Einbettung von Studien(fach)wahlen entsteht eine Nähe zum Studium der Sozialen Arbeit unter spezifischen Einflüssen, die sowohl die gesellschaftliche Arbeitsteilung qua Geschlecht umfassen wie auch die Nähe zur Sozialen Arbeit im Rahmen bestimmter Milieuorientierungen: Zum einen zeichnet sich die grundsätzliche Tendenz ab, dass die Dispositionen der selbstbestimmteren Milieus zu diesem Studienfach ,passen', insbesondere, wenn sie von einer Distanz zu Status- und Prestigedenken geprägt sind. ${ }^{3}$ Gerade bei Männern scheint eine Abkehr von erwerbsbezogener Leistungsorientierung maßgeblich dafür, dass sich ein Weg zur Sozialen Arbeit hin öffnet: Diese Abkehr scheint ein Türöffner dafür zu sein, dass ,Männlichkeit' stärker mit ,Fürsorge' verknüpft wird als mit einer versorgungsund erfolgsorientierten Funktion. Auch eine Nähe zu kommunikativen Lehrund Lernformen auf Augenhöhe und zu kontroversen Aushandlungsprozessen

${ }^{3}$ Dass nach der Motivforschung bei Studierenden mit pädagogischem Interesse das Motiv von „Selbstfindung und -entwicklung“ besonders bedeutsam ist (Bohrmann/Gehrmann/Klein 2002), lässt sich vor diesem Hintergrund als milieuspezifische Nähe zur sozial-pädagogischen Fachkultur in den selbstbestimmteren Milieus deuten. 
macht die Wahl eines sozialarbeiterischen Studiums sowohl für Frauen wie auch für Männer wahrscheinlicher und legt einen Grundstein für die Nähe zur sozialpädagogischen Fachkultur. Außerdem begünstigen Erfahrungen von Notwendigkeiten, Schullaufbahnen mit Umwegen und Hürden und gemeinschaftliche und/oder egalitäre Handlungsorientierungen einen Einstieg in die Soziale Arbeit. Hinzu kommt, dass diese Habitusmuster milieuübergreifend eine höhere Passfähigkeit zu weiblich-vergeschlechtlichten Habitus haben, die sich weniger an stringentem Karrierestreben und berufsbezogenem Prestige ausrichten als bei den Männern des gleichen Milieus - so werden auch die Wege in die Soziale Arbeit häufig von weiblichen Orientierungspersonen und einer stärkeren Bedeutung der Mutter flankiert. Allerdings ist die Studierendenschaft innerhalb einer Fachkultur, so auch in der Sozialen Arbeit, diesen Tendenzen zum Trotz nicht homogen: So kann etwa die Deutung der Sozialen Arbeit je nach Milieu stark differieren in einem Kontinuum vom idealistischen Blick auf den Beruf als „Stachel im Fleisch der herrschenden Systeme“ (gefunden beim Muster „Idealismus und Unkonventionalität") bis zum leistungs- und wettbewerbsorientierten Verständnis als Instanz zur „Umstellung und Erziehung“ (gefunden beim Muster „Aufstiegsstreben und Disziplin“).

Eine Nähe zum Studium des Bauingenieurwesens wird wiederum unter anderen spezifischen Einflüssen wahrscheinlicher: So deutet sich eine höhere Passung in den disziplinierten und hierarchisch orientierten Milieus an und ebenso bei einer ausgeprägten Erwerbsarbeits- und Leistungsorientierung. ${ }^{4}$ Eine Disposition hin zu klaren Strukturen wiederum lässt Parallelen erkennen zu einer mathematischen Affinität und zur ingenieuralen Fachkultur mit ihrer hohen Bedeutung von Faktenwissen und einem nüchternem Anwendungsbezug. Dazu gehört in einigen Fällen auch eine fachliche Begeisterung für das tüftlerische Rechnen, Werken, für das Handwerkliche oder für mathematisch-theoretische Fragestellungen. Diese fachliche Affinität allein bzw. isoliert ist nicht ausschlaggebend für eine technische Studienfachwahl, sondern kommt in Verbindung mit den milieuspezifischen Strategien zum Wirken. So führt eine Ausbildung im handwerklich-technischen Bereich und die handwerkliche Affinität dann nicht zu einem technischen Studium, wenn eine geringe Passung zu einem Ingenieurberuf vorhanden ist - etwa aufgrund einer großen Distanz zu den entsprechenden fachkulturellen Lehr- und Lernformen, einer vergleichsweise geringen Orientierung an Erwerbsarbeit und einer Abgrenzung von einem diszipliniertem Vorgehen. Vor dem Hintergrund

\footnotetext{
${ }^{4}$ Analog dazu lässt sich die Erkenntnis der Motivforschung deuten, dass für Studierende der Ingenieurwissenschaften die Verdienstmöglichkeiten und der Status des Berufs besonders wichtig sind (Scheller/Isleib/Sommer 2013).
} 
milieuspezifischer Strategien kann auch das Studium des Bauingenieurwesens wiederum unterschiedliche Zwecke erfüllen - etwa zum Erlangen von mehr (beruflicher) Autonomie (gefunden beim Muster „Autonomie durch Leistung“), zur Festigung des familiären Status (gefunden bei den Mustern „Aufstiegsstreben und Disziplin“ und „Traditions- und Stabilitätsorientierung“) und/oder zur Wahrung der familiären Tradition (gefunden beim Muster ,Traditions- und Stabilitätsorientierung“).

\section{Vergeschlechtlichte Passungsverhältnisse}

Umgekehrt muss keine ,techniknahe Sozialisation" vorhanden sein, um den Weg in ein Bauingenieurstudium zu ebnen - zumindest nicht bei einer männlichvergeschlechtlichten Sozialisation, wie sich beim Muster von ,Disziplin und Erwerbsorientierung“ zeigt: Im Rahmen eines patriarchal vererbten Auftrags zur familiären Statussicherung auf der Basis von Erwerbsarbeit kann ein Technikstudium auch ohne familiär angelegte fachliche Nähe ,Mittel zum Zweck` sein und durch erwerbs- und leistungsorientierte sowie disziplinierte Habitusmuster und eine institutionelle Kanalisierung - wie die Vermittlung in eine technische Berufsausbildung - wahrscheinlich werden. Vergeschlechtlichte Abdrängungsmechanismen im mathematisch-technischen Bereich wiederum zeichnen sich nur bei den ,weiblichen" Fällen ab, verhindern aber die Wahl eines entsprechenden Studiums bei diesen Bauingenieurstudentinnen nicht, weil die vorhandenen Milieuorientierungen entsprechend bestärkend wirken.

Die Vorstellungen von der Familiengründung wiederum sind bei allen Fällen von den Erfahrungen in der Herkunftsfamilie geprägt und verwoben mit den vorhandenen Milieuorientierungen, sodass die antizipierte Familiengründung etwa besonders von den Werten von Gemeinschaft und Zusammensein geprägt sein kann oder - als Kontrast - von Fragen des Status auf der Basis einer familiären unternehmerischen Einheit. Im Sample sind die Familienvorstellungen durchgehend heteronormativ geprägt, sodass bei den Befragten ausschließlich von heterosexuellen Paarbildungen ausgegangen wird und sich zudem milieuübergreifend eine vorreflexive Zuständigkeit für Familienarbeit auf weiblicher Seite abzeichnet - allerdings in unterschiedlicher Ausprägung je nach Milieu und Geschlecht. ${ }^{5}$ Dabei muss eine als unkonventionell konstruierte Studien(fach)wahl nicht mit unkonventionellen Vorstellungen von Familiengründung und -organisation einhergehen, wie es sich im Muster von „Unkonventionalität

\footnotetext{
${ }^{5}$ Diese Varianz zwischen Frauen (bzw. zwischen Männern), was ihre Vorstellungen von Familiengründung und -organisation angeht, und die gleichzeitige Resistenz der intrafamiliären Arbeitsteilung gegenüber Modernisierungsprozessen, spiegeln sich auch bspw. in den Ergebnissen der Shell-Studien wieder (Geissler/Oechsle 2002).
} 
und Idealismus“ abzeichnet. Familienvorstellungen mit mehr egalitärem Potential finden sich beim Muster ,Gemeinschaft und Ordnung“, da die Familienarbeit hier als gemeinsame Aufgabe gedeutet wird. Die hohe Erwerbsorientierung im Muster „Erwerbsorientierung und Disziplin“ wiederum führt zur antizipierten Übernahme der Versorgerrolle auf männlicher Seite und zum Spannungspotential auf weiblicher Seite, wenn die Zuständigkeit für Familienarbeit zugleich antizipiert und abgewertet wird. Notwendigkeitserfahrungen und Sicherheitsorientierung - etwa beim Muster „Autonomie durch Leistung“ - können wiederum zur Antizipation einer traditionellen Aufgabenteilung aus monetären und pragmatischen Gründen führen. Das gleiche Resultat, aber mit anderem habitusspezifischen Mechanismus, sieht man schließlich beim Muster „Traditions- und Stabilitätsorientierung“, bei dem eine affektive Zuschreibung dichotomer Zuständigkeiten und Eigenschaften nach Geschlecht am stärksten verankert zu sein scheint und bei dem sich kein Spannungspotential auf weiblicher Seite andeutet.

Wie die Passung zu einem Studienfach und perspektivisch dem daran anschlieBenden Berufsfeld mit der Vorstellung von Familiengründung verknüpft ist, schlägt sich besonders bei den männlich-vergeschlechtlichten Fällen nieder: Milieuübergreifend orientiert sich keine der weiblichen Befragten an einer erwerbsorientierten Versorgerinnenfunktion, jedoch findet diese Orientierung in unterschiedlichem Ausmaß bei fast allen der männlichen Fälle statt. Damit diese Orientierung trotzdem die Wahl der Sozialen Arbeit als Beruf mit wenig Aufstiegs- und Gehaltsaussichten zulässt, darf sie erstens nicht besonders ausgeprägt sein und es müssen zweitens offenbar weitere Bedingungen erfüllt sein wie die positive Konnotation pädagogischer Tätigkeiten und die Habitualisierung in einem Milieu jenseits von Prestige- und Statusdenken und/oder mit einer hohen Bedeutung von Gemeinschaft. Die Wahl eines Studienfachs findet bei den männlich-vergeschlechtlichten Fällen so vor dem Hintergrund einer Passung von Fach- und Berufskultur und der milieuspezifischen Männlichkeitskonstruktion statt. Umgekehrt scheint die Studienfachwahl von Frauen weniger beeinflusst zu sein von ihren Vorstellungen von Familiengründung: Es schreiben sich in der Sozialen Arbeit wie im Bauingenieurwesen alle weiblichen Befragten tendenziell die Zuständigkeit für Familienarbeit zu. Wie stark diese Zuständigkeit ausgeprägt ist und warum sie antizipiert wird - etwa aus einer Distanzierung von einem Erwerbsfokus heraus oder aus monetären und pragmatischen Gründen - ist wiederum milieuspezifisch gefärbt. Diese milieuspezifischen Weiblichkeitskonstruktionen scheinen aber nur lose mit der Studienfachwahl verbunden zu sein, denn ,traditionelle' Vorstellungen von Familienorganisation sind in beiden Fachkulturen zu finden und führen weder bei der einen noch der anderen Fachwahl zu einem ausgeprägten Spannungspotential. Während das Milieu also für den Weg 
von Männern in die Soziale Arbeit entscheidend zu sein scheint, wird der Weg von Frauen in das Bauingenieurwesen stärker - aber natürlich nicht milieuunabhängig - von anderen Einflüssen geprägt wie Orientierungspersonen und Elementen der fachkulturellen Sozialisation.

Zusammengefasst sind diese Analyseergebnisse zu milieuspezifischen Studien(fach)wahlen, zur entsprechenden Nähe zu einem Studiengang bzw. einer Fachkultur deren Einbettung in die Lebensführung mit einem Fokus auf die vergeschlechtlichte Arbeitsteilung in Tabelle 8.1. Die Muster sind nach dem tendenziellen Ausmaß ihrer Passung zu den beiden Fachkulturen angeordnet: Vom Muster von „Idealismus und Unkonventionalität“ links (bei dem sich die größte Nähe zur Sozialen Arbeit abzeichnet) bis zum Muster der „,Traditions- und Stabilitätsorientierung“ rechts (dessen Dispositionen besonders zur ingenieuralen Fachkultur passen). Dazwischen befinden sich die Muster von „Gemeinschaft und Ordnung“ (das aufgrund der ausprägten gemeinschaftlichen Orientierung eine Nähe zur Sozialen Arbeit bedingt), ,Autonomie durch Leistung“ (in dem im Sample sowohl die Soziale Arbeit wie auch das Bauingenieurwesen ein Schritt hin zum Bildungsaufstieg ist) und „Aufstiegsstreben und Disziplin“ (in dem eine ingenieurale Studienfachwahl aufgrund der hohen Bedeutung von Status näher liegt, aber in dem - zumindest im weiblich-vergeschlechtlichten Fall - auch ein Weg hin zur Sozialen Arbeit mit einer leistungsorientierten Deutung der Profession möglich ist). So entsteht das Gesamtbild der Passung zu einer Fachkultur bzw. einem Beruf, innerhalb der die Studien(fach)wahl eingebettet ist in die milieuspezifische Lebensführung und die ebenso milieuspezifischen Geschlechterpraktiken.

Diese Erkenntnisse und die vorangegangenen Fallanalysen können nur ein erster Schritt sein hin zur Entschlüsselung der sozialen Logiken von vergeschlechtlichten milieuspezifischen Studien(fach)wahlen. Sie zeigen exemplarisch, wie die Nähe zu einem Studienfach im Zusammenspiel von sozialem Milieu und Geschlecht entsteht und dass die Betrachtung nur einer dieser Dimensionen dem komplexen Prozess der Studien(fach)wahl nicht gerecht würde.

\subsection{Rückbindung an den Diskurs der Geschlechterforschung}

Die vorliegende Arbeit ergänzt die bisherigen Studien der Geschlechterforschung zu Studien(fach)wahlen und verweist auf die Notwendigkeit eines systematischen Zusammendenkens von Bildungs- und Geschlechterforschung bzw. von sozialer 


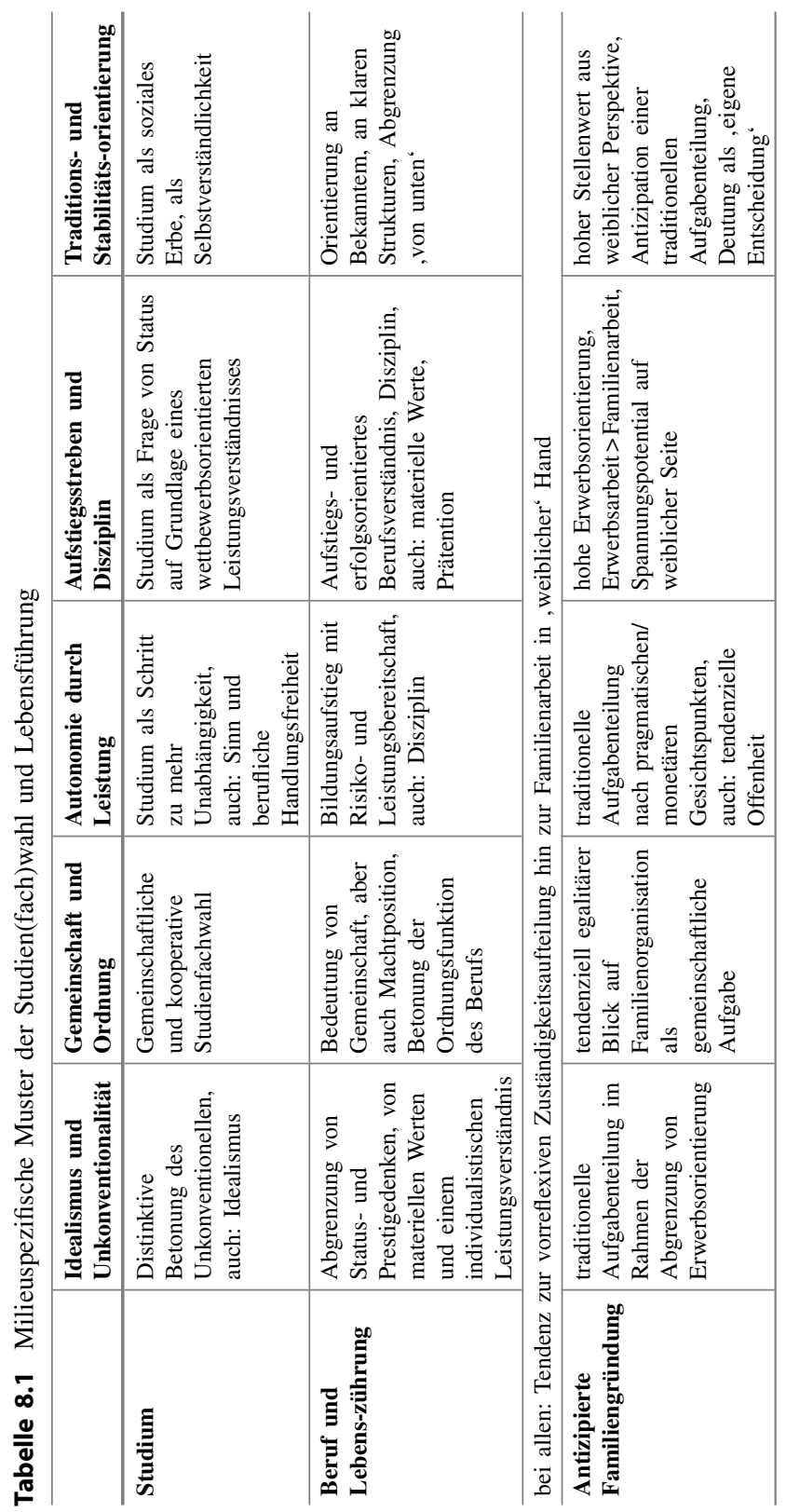


Klasse und sozialem Geschlecht. An die entsprechenden Forschungsstände und Diskurse werden die Ergebnisse nun rückgebunden.

Dieses Zusammendenkens bedarf es erstens auf der Ebene der Bildungsentscheidungen bzw. Studien(fach)wahlen, bei der aus Perspektive der Geschlechterforschung auch systematisch die soziale Herkunft berücksichtigt werden muss. So sind sämtliche prägende Einflüsse von Geschlecht - seien es Geschlechterstereotype (Makarova/Aeschlimann/Herzog 2016; Schmirl et al. 2012), die gleichzeitige und hierarchisierende Konstruktion von Beruf und Geschlecht (Wetterer 2002) und der damit verbundene kulturell geformte Ausschluss des Sozialen aus dem technischen Berufsfeld (Schmeck 2019), die spezifischen Ressourcen in der (techniknahen) Sozialisation (Schüller/Braukmann/Göttert 2016; Wensierski/Langfeld/Puchert 2015; Puchert 2017) oder der Zusammenhang von antizipierter Familiengründung und Fachkultur bzw. Berufswahl (Haffner 2014; Wehner et al. 2016; Becker-Schmidt 2008) - eingebunden in und untrennbar verwoben mit der Habitualisierung in einem bestimmten sozialen Milieu.

Durch das Habituskonzept wiederum wird ein systematischer Blick auf die durch die soziale Herkunft bedingten Differenzen innerhalb einer Geschlechtskategorie eröffnet: Demnach kann der Weg in ein Ingenieurstudium ein selbstverständlicher zur Wahrung der familiären Tradition und mit vergleichsweise geringen vergeschlechtlichten Abdrängungseffekten sein, wie es sich im oberen Bereich der ständisch-kleinbürgerlichen Traditionslinie abzeichnet. Oder ein Ingenieurstudium kann im Fall eines Bildungsaufstiegs in der Traditionslinie von Facharbeit und praktischer Intelligenz mit Anstrengungen und Unsicherheiten verbunden sein und weniger den Zweck der Traditionswahrung und Statussicherung erfüllen als eher auf mehr (berufliche) Autonomie abzielen. Der Weg von Männern in einen sozialen Beruf wiederum scheint insbesondere in den selbstbestimmteren Milieus mit gemeinschaftlichen Handlungsorientierungen nahezuliegen - denn hier engen weder ein status- und prestigeorientierter Erwerbsfokus noch die Antizipation einer familiären Versorgerrolle die männlichvergeschlechtlichte Berufswahl ein.

Zweitens sind die Dimensionen von Klasse und Geschlecht in Fragen der Familiengründung und -organisation eng verknüpft und ziehen weitreichende Folgen mit sich: So finden sich in den Fallanalysen und entsprechenden milieuspezifischen Mustern der Studien(fach)wahl Hinweise auf die Ursachen für die vertikale Unterschichtung von Frauen je Milieu (Vester/Gardemin 2001): Die heterosexuelle Paarbildung scheint auch im vorliegenden Sample tendenziell den 
Logiken von Klassenhomogamie und Habitusähnlichkeit ${ }^{6}$ (Frerichs 2000, Steinrücke 2006) sowie dem von Bourdieu benannten „weiblichen Masochismus“ zu folgen (Bourdieu 1997b: 229 f.). Fasst man diesen „weiblichen Masochismus“ nicht wie Bourdieu als Paarbildung von Frauen mit Männern in Machtpositionen, sondern etwas abgeschwächter als Paarbildung mit Männern in vergleichsweise höheren sozialen Positionen, so erklärt sich die Reproduktion eines hierarchischen Geschlechterverhältnisses je Milieu. Zusätzlich bilden diese Mechanismen heterosexueller Paarbildung die Grundlage für eine monetäre Begründung von traditionellen Arrangements der Arbeitsteilung, was die Hierarchie des Geschlechterverhältnisses um ein weiteres verfestigt. Diese monetär bedingte Verfestigung trifft besonders diejenigen Milieus und ihre Angehörigen, bei denen finanzielle Absicherung und Erfahrungen finanzieller Notwendigkeit eine (tragende) Rolle spielen - bei denen also die soziale Position eine eher unsichere und abzusichernde ist, weshalb im Kontext hierarchischer Gehaltsverhältnisse zwischen beiden Partner*innen das niedrigere Gehalt fast automatisch mit der Übernahme von Familienarbeit verbunden ist. Spannungspotential wiederum bergen die vergeschlechtlichten Praktiken der Arbeitsteilung vor allem für die weiblichen Milieuangehörigen in den leistungs- und aufstiegsorientierten Milieus, wenn der milieuspezifische Glaube an Leistungsgerechtigkeit und das Streben nach beruflichem Aufstieg vor dem Hintergrund ihrer Zuständigkeit für Reproduktionsarbeit möglicherweise nicht erfüllbar sind. In den ständisch-kleinbürgerlichen Milieus spiegelt sich dieses Spannungspotential weniger wider, da hier auch auf weiblicher Seite eine stärkere Anlehnung an die bekannte binäre Arbeitsteilung stattfindet.

Die von Koppetsch festgestellte „Brückenfunktion“ (Koppetsch/Burkart 1999: 13) von Geschlecht als konstitutiv für Klassenverhältnisse bestätigt sich damit in doppelter Weise: zum einen in Gestalt einer vergeschlechtlichten sozialen Platzanweisung im Prozess der Studien- und Berufswahl und zum anderen in Form der hierarchisierenden Mechanismen von Familienorganisation. Diese milieuspezifischen und zugleich milieukonstituierenden Elemente der Vergeschlechtlichung lassen sich als zwei Dimensionen der Habitusdimension von Geschlecht fassen und sie illustrieren das untrennbare und interdependente Zusammenspiel von Geschlechts- und Klassenverhältnissen in der Produktion sozialer Ungleichheit.

Die herausgearbeiteten Muster der Studien(fach)wahl und damit verknüpften Lebensführung entspannen sich somit entlang von acht Einzelfällen, weisen aber aufgrund der Hintergrundfolie sozialer Milieus zugleich über diese Einzelfälle

\footnotetext{
${ }^{6}$ Wie etwa gerade bei den Erzählungen von Michael über seine Partnerin und bei Jennifer über ihren Partner deutlich wird.
} 
hinaus auf übergeordnete Mechanismen sozialer Ungleichheit im Kontext von sozialer Herkunft, Geschlecht, Beruf und Arbeitsteilung.

\subsection{Rückbindung an den Diskurs der Bildungsforschung}

Die Rückbindung an den Diskurs der Bildungsforschung soll primär anhand der beiden bereits nachgezeichneten dominanten Paradigmen nach Boudon und Bourdieu erfolgen. Die vorliegende Arbeit zeigt, wie eine an Bourdieu angelegte Untersuchung vergeschlechtlichter Studien(fach)wahlen über die Boudonsche Perspektive hinausgehen kann: Indem nicht von a priori festgelegten binären ,Geschlechtereffekten' ausgegangen wird, können stattdessen die habitusspezifischen Herstellungsprozesse von Geschlecht in den Blick genommen werden. So wurde gezeigt, wie etwa Männlichkeit in unterschiedlichen Milieus konstruiert und praktiziert wird und dass die Sozialisation in einem gemeinschaftsorientierten Milieu jenseits von Aufstiegsstreben und materiellen Leitwerten eine fürsorgliche Männlichkeitspraxis und eine entsprechende sozialpädagogische Fachwahl hervorbringen kann. Die Analyse ebendieser Prozesshaftigkeit vorreflexiv angelegter Bildungsentscheidungen im Kontext von sozialer Herkunft und Geschlecht ist eine der Stärken der vorliegenden Arbeit.

Darauf aufbauend lässt sich die von Lojewski (2012) vorgenommene und an Boudon angelehnte These, in mittleren und höheren sozialen Schichten herrschten fortschrittlichere Haltun gen zur Arbeitsteilung vor und lägen entsprechend einkommens- und aufstiegsträchtigere Studienfachwahlen näher, durch die durchgeführten Analysen widerlegen: Es wurde erstens gezeigt, dass die Orientierung an Einkommen, materiellen Werten, individualistischer Leistung und Aufstiegsstreben weniger einer Frage, vertikaler' Verortung ist, sondern vielmehr das Resultat der Habitualisierung in einem spezifischen sozialen Milieu - im vorliegenden Sample durch das Muster von „Aufstiegsstreben und Disziplin“ vertreten. Die entsprechenden aufstiegs- und konkurrenzorientierten Habitusmuster wiederum wirken dabei in vergeschlechtlichtem Ausmaß, wodurch Männern dieser Milieus eine weniger aussichtsreiche Studien(fach)wahl wie die zur Sozialen Arbeit noch ferner zu liegen scheint als den Frauen ähnlicher sozialer Positionen. Zweitens verdeutlichen die Fallanalysen, dass auch die Einstellung zur Arbeitsteilung keiner, vertikalen' Logik folgt. Vielmehr schlagen sich hier ebenso beträchtliche horizontale Unterschiede nieder. Zudem scheinen die Vorstellungen von Arbeitsteilung ausschließlich bei den männlichen Befragten in einem engen Zusammenhang mit der Studien(fach)wahl zu stehen. Ein vergleichbarer Zusammenhang von antizipierter Familiengründung und Studien(fach)wahl zeichnet sich bei den weiblichen Fällen nicht ab. 
Schließlich bietet sich noch ein Vergleich der Ergebnisse dieser Untersuchung mit Studien ähnlicher theoretischer und methodologischer Ausrichtung an, die ebenso die Gruppe von Studierenden in den Blick genommen haben (etwa LangeVester/Teiwes-Kügler 2004; Grunau 2017; Hild 2019). Zwar haben diese Studien einen anderen Fokus (nämlich die Praktiken von Studierenden statt ihrer Studienfachwahlen) und betrachten andere Fachkulturen, allerdings schlägt sich die grundsätzliche Struktur des Raums der sozialen Milieus auch dort in ähnlicher Weise nieder.

So sind Parallelen festzustellen zwischen den Mustern der Studierpraktiken und den Mustern der Studien(fach)wahl von Studierenden ähnlicher sozialer Positionen. Die vorliegende Arbeit bestätigt und ergänzt diese Studien damit zum einen durch ihren Fokus auf die Fachwahl und zum anderen durch die besondere Betrachtung der Habitusdimension von Geschlecht. Beispielhaft sei dies anhand von zwei Vergleichen verdeutlicht:

Der Studierendentyp der „Effizienzorientierten“ nach Lange-Vester/TeiwesKügler mit einem strebenden Habitus, der mit Anstrengung und hoher Leistungsfähigkeit verbunden ist (Lange-Vester/Teiwes-Kügler 2004: 177) zeigt zum einen Parallelen zum ,aufstiegsorientierten Typus“ nach Grunau mit seinen ,strebenden“" und „leistungsorientierten“ Handlungsmustern (Grunau 2017: 177 ff.) und mit dem Muster der Studien(fach)wahl von „Aufstiegsstreben und Disziplin“. Die drei ,Typen“ sind zwar nicht deckungsgleich im Raum der sozialen Milieus verortet - schließlich unterscheiden sie sich in Feinheiten, ebenso wie sich das Sample unterscheidet - aber in einem ähnlichen Bereich des Modells. Während die Studien von Lange-Vester/Teiwes-Kügler und Grunau die entsprechenden Studierpraktiken herausarbeiten, zeigt die vorliegende Arbeit, wie auch die Studien(fach)wahl in diese Habitusmuster eingebettet ist. Die Studentin und der Student dieses Musters sind dabei verbunden durch ihre ähnlichen habitusund milieuspezifischen Muster, aber zugleich zeichnen sich Elemente der Vergeschlechtlichung ab: Die ausgeprägte Orientierung an materiellen Werten, Status und beruflichem Erfolg scheint die ,männliche' Studien(fach)wahl stärker zu begrenzen als die ,weibliche' und verleiht ihr zugleich das Privileg, einem aussichtsreichen Ingenieurfach näher zu sein. Auf weiblicher Seite wiederum kann eine prestigearme, sozialpädagogische Studien(fach)wahl nur als ,Zwischenstation' gesehen werden oder dem sozialen Umfeld gegenüber erklärungsbedürftig sein. Außerdem führt die dominante Aufstiegs- und Leistungsorientierung auf weiblicher Seite zu einer Spannung hinsichtlich der antizipierten Erziehungsarbeit nach Familiengründung.

Die ,genügsame“ Fraktion der „Ganzheitlichen“ nach Lange-Vester/TeiwesKügler wiederum (2004: 175 ff.) zeigt Schnittstellen mit dem Muster der 
Studien(fach)wahl von „Autonomie durch Leistung“: Auch hier geht es um einen begrenzten sozialen Aufstieg und das Ergreifen eines Berufs mit mehr Handlungsfreiheit und Selbstständigkeit. Die vorliegende Arbeit geht durch ihren Fachvergleich noch einen Schritt weiter und zeigt, wie im Rahmen dieses Habitussyndroms unterschiedliche Fachwahlen zustande kommen können: So ist es die spezifische Kombination aus gemeinschaftlichen Dispositionen und Erfahrungen in fürsorglichen Tätigkeiten, die die Wahl eines sozialpädagogischen Studiums ebnet. Umgekehrt können (familiäre) Einblicke in einen technischen Beruf den Weg eröffnen, entsprechende Fachinteressen zu wecken und sich einen sozialen Aufstieg in diesem Feld zuzutrauen.

Damit ergänzt die vorliegende Arbeit vorhandene Habitusuntersuchungen unter Studierenden dadurch, dass sie die spezifischen milieu- und geschlechtsspezifischen Passungsverhältnisse beleuchtet, auf denen die späteren Studierpraktiken aufbauen.

\subsection{Ansätze für Praxis und Forschung}

Die dargestellten Ergebnisse haben für mehrere Praxisbereiche Relevanz: Erstens ermöglicht die Analyse von milieuspezifischen Wegen in ein Studium ein differenziertes Bild über die Zielgruppe der Studienberatung. Mit einem detaillierten und ungleichheitssensiblen Wissen darüber, wie die Studienaufnahme durch die soziale Herkunft geprägt ist, kann unter den Stichworten der „Habitussensibilität“ (Sander 2014) und der „Habitus-Struktur-Reflexivität“ (Schmitt 2014) fundiert beraten und es können möglicherweise verschiedene Ungleichheitsmechanismen abgemildert oder sie können zumindest in einem ersten Schritt reflektiert und bewusst gemacht werden. Mit Rückgriff auf das Habituskonzept können außerdem die Dimensionen von Geschlecht und sozialer Herkunft gleichermaßen auch in der Beratung berücksichtigt werden - um etwa einer Homogenisierung von Frauen in Ingenieurfächern zu entgehen und sie in ihrer milieuspezifischen Einbettung und den damit verbundenen strukturellen Abdrängungen und/oder Privilegien zu sehen.

Damit erlauben die Ergebnisse zweitens auch Orientierungsprojekten für Frauen im MINT-Bereich ein ungleichheitssensibleres Arbeiten mit der Zielgruppe, indem die soziale Herkunft systematisch mitgedacht wird. Es darf nicht nur darum gehen, Geschlechterstereotype in der Schule aufzubrechen, Schülerinnen (weibliche), Role Models`zur Verfügung zu stellen oder Frauen mit technischem Berufsinteresse miteinander $\mathrm{zu}$ vernetzen, sondern diese Maßnahmen müssen auch gleichzeitig auf eine breite soziale Herkunft unter den 
künftigen Studentinnen abzielen. Wie sich in der vorliegenden Arbeit andeutet, kann der Bildungsaufstieg im Sinne einer teilweisen Abkehr von den Normen und Werten der Herkunftsfamilie dadurch unterstützt werden, dass er gemeinsam mit anderen Personen einer ähnlichen sozialen Herkunft vorgenommen wird wie Peers, Partner*innen oder Geschwistern. Maßnahmen zur Vernetzung von Schülerinnen mit Technikinteresse könnten also um eine herkunftssensible Komponente ergänzt werden, indem diese Vernetzung Schülerinnen einer ähnlichen sozialen Herkunft zusammenbringt - und ihnen womöglich noch , Role Models" mit einem vergleichbaren sozialen Hintergrund zur Verfügung stellt. Damit könnten ,zwei Fliegen mit einer Klappe‘ geschlagen und Kinder aus Nicht-Akademiker*innenfamilien zu einem Studium allgemein und Frauen zu einem technischen Studienfach ermutigt werden. Diese ,Doppelorientierung ' wird in aktuellen MINT-Projekten bislang kaum berücksichtigt. Dass junge Frauen aus Familien ohne Hochschulerfahrung hier wiederum nochmals horizontal zu differenzieren sind nach der alltagskulturellen Praxis ihrer Herkunftsfamilien, ermöglicht den sensiblen Zugang auf die Zielgruppe um ein weiteres.

Zugleich ist die Hoffnung auf Umwälzung berufsbezogener Herrschaftsverhältnisse durch einen steigenden Frauenanteil in Ingenieurberufen eine vage denn mit Rückgriff auf Wetterer (2002) und Bourdieu (2005) wäre dann eher mit einer Intensivierung der intraberuflichen Geschlechterhierarchie sowie dem allgemeinen Statusverlust des Berufsfeldes zu rechnen, solange die dahinter liegende Trennung von Weiblichkeit und Männlichkeit durch einen „negativen symbolischen Koeffizienten“ (ebd.: 161 f.) bestehen bleibt. Erst eine Umkehr dieses symbolischen Machtverhältnisses könnte eine tatsächliche Angleichung der sozialen Position von Frauen und Männern bewirken - eine Vorstellung, deren Utopie unter den aktuellen gesellschaftlichen Verhältnissen nicht zu leugnen ist. Das dagegen pragmatische Ziel, mehr Frauen für technische Berufe zu gewinnen, mag daher ein notwendiger, da realistischer Schritt hin zu gleichen Geschlechterverhältnissen sein - und zugleich darf er nicht der letzte sein.

Was die Forderung nach entsprechenden Orientierungsprojekten für Männer in sozial(pädagogisch)en Berufen betrifft, wäre ein solches ,pragmatisches Ziel ${ }^{*}$ der Erhöhung des Männeranteils möglicherweise zu kurz gedacht. Es müssten noch weitere Faktoren berücksichtigt werden: Schließlich wird diese Forderung häufig vor dem Hintergrund der bereits erwähnten „,binär-geschlechtliche Ganzheitsfigur" (Rose 2014: 30) gestellt. Es wird also davon ausgegangen, Kinder bräuchten ,männliche 'Vorbilder, an die zugleich stereotype Erwartungen gestellt werden: So werden dichotome Zuschreibungen an Pädagogen aktiviert, durch die „Väterlichkeit als Kompensation für Kinder alleinerziehender Mütter [gilt], Sportlichkeit als Ausdruck männlichen Raufens und aggressives Durchsetzen von 
Stärke" (Faulstich-Wieland 2012: 28). Dabei zeigt auch die vorliegende Arbeit, dass und warum gerade jene Männer in sozial(pädagogische)Berufe gehen, die eben nicht der binären Vorstellung von einer ,stereotypen' bzw. ,hegemonialen' Männlichkeit entsprechen: Der Weg in einen sozialen Beruf liegt nämlich insbesondere Männern aus sozialen Milieus mit gemeinschaftlichen, egalitären Handlungsorientierungen jenseits von Status- und Prestigedenken nahe, auf die dichotome Zuschreibungen weniger zutreffen mögen als auf Männer mit einem eher individualistischen, wettbewerbs- und statusorientierten Habitus. Diesen Umstand an sich als defizitär zu bewerten, wäre eine fatale Herabsetzung der entsprechenden milieuspezifischen Männlichkeitspraktiken. Umgekehrt mag es jedoch eine pädagogisch und geschlechtertheoretisch sinnvolle Forderung sein, vielfältige Männlichkeitspraktiken in sozial(pädagogisch)en Berufen abzubilden. Dieses Ziel wiederum verweist auf den notwendigen Abbau des symbolischen Geschlechterverhältnisses, um die Passung diverser (klassenspezifischer und) vergeschlechtlichter ${ }^{7}$ Habitus zu ermöglichen. Orientierungsprojekte für Männer in sozialen Berufen müssten also auf die Umwertung ,sozialer' Berufe abzielen und zugleich auf die berufliche Wertschätzung vielfältiger vergeschlechtlichter Habitus hinwirken. Werden diese Faktoren nicht berücksichtigt, würden entsprechend unreflektierte Orientierungsprojekte wohl - wenn überhaupt - nur zu einem Anstieg spezifischer Männlichkeiten in sozial(pädagogisch)en Berufen führen und es würde weiterhin zu intraberuflichen Segregationsprozessen kommen. Beides ist weder aus pädagogischer noch aus gleichstellungspolitischer Sicht erstrebenswert.

Und drittens sind die Ergebnisse zuträglich für die untersuchten Professionen selbst, wie sich am Beispiel der Sozialen Arbeit zeigen lässt: Wenn die Soziale Arbeit etwa derart milieuspezifisch von ihren Studierenden und künftigen Sozialarbeiter*innen gedeutet werden kann, dass sie als „Stachel im Fleisch der herrschenden Systeme“ oder als Instanz zur „Umstellung und Erziehung“ gilt, impliziert das einen je spezifischen Zweck der Profession und eine spezifische Sicht auf das Verhältnis zur Klientel. Hier bedarf es einer professionellen Selbstreflexion, um sich der eigenen habitusspezifischen Deutungsmuster zumindest teilweise bewusst zu werden, sie mit den bestehenden Strukturen abzugleichen und so Leerstellen im eigenen Handeln aufzudecken.

Auch für die Forschung bieten sich mehrere Anknüpfungspunkte: Um die sozialen Logiken hinter Studien(fach)- und Berufswahlen noch tiefer herauszuarbeiten, ist die stärkere Verknüpfung der Erkenntnisse aus Geschlechter- und Bildungsforschung notwendig, wie sie hier begonnen wurde. Der explorative

${ }^{7}$ auch weiblicher 
Schritt, der mit dieser Arbeit getan wurde, könnte ergänzt werden um das Herausarbeiten weiterer milieuspezifischer Muster und ihrer Vergeschlechtlichung - bei mehr Fällen und mehreren Fachkulturen, um neue Vergleiche zu ermöglichen und die Aussagekraft der gefundenen Muster zu erhöhen.

Des Weiteren wäre ein solcher Blick auf vergeschlechtlichte milieuspezifische Passungsverhältnisse nicht nur am Übergang in das Studium interessant, sondern auch gerade im weiteren Verlauf: Welche Habitustypen werden etwa aus den Studiengängen von Sozialer Arbeit und Bauingenieurwesen abgedrängt und welche haben eine besonders hohe Passfähigkeit? Wie kommen hier die Habitusdimensionen von Geschlecht und Milieu zusammen? Finden bestimmte Typen den Einzug in bestimmte Berufsfelder und was bedeutet das für diese Felder? Diese Fragen verweisen damit auf die Notwendigkeit entsprechender Längsschnittuntersuchungen, die zeitlich über die in der vorliegenden Arbeit untersuchten ,antizipierte Passung' hinausgehen und die Passungsverhältnisse im Studienverlauf in den Blick nehmen.

Außerdem weist die vorliegende Arbeit dringend darauf hin, dass Geschlecht in Studien der Bildungsforschung nicht nur als quantitatives Verteilungsverhältnis behandelt werden darf, wie es häufig noch der Fall ist, sondern dass dieser quantitative Ansatz um die Frage nach den Herstellungsprozessen von Geschlecht ergänzt werden muss. Umgekehrt bedarf es in der Geschlechterforschung noch stärker der systematischen Berücksichtigung der sozialen Herkunft, die zumeist gar nicht oder nur im Sinne einer vertikalen Einteilung in Berufs- und Bildungsschichten eingearbeitet wird. Hierzu regt das Vorgehen dieser Arbeit an, das „,intersektionale Potential“ des Habituskonzepts (ebd.) noch weiter auszureizen: Die Habitushermeneutik wurde hier erstmalig fokussiert auf die Dimension von Geschlecht im Kontext sozialer Milieus angewendet und diese methodologische Erprobung ist an dieser Stelle keinesfalls abgeschlossen. An diesem Punkt weiterzuarbeiten, um das Zusammenwirken von Geschlecht, Milieu - und anderen Dimensionen sozialer Praxis - habitushermeneutisch zu entschlüsseln, ist ein vielversprechender Anstoß für die Zukunft von Geschlechter- und Bildungsforschung. 
Open Access Dieses Kapitel wird unter der Creative Commons Namensnennung 4.0 International Lizenz (http://creativecommons.org/licenses/by/4.0/deed.de) veröffentlicht, welche die Nutzung, Vervielfältigung, Bearbeitung, Verbreitung und Wiedergabe in jeglichem Medium und Format erlaubt, sofern Sie den/die ursprünglichen Autor(en) und die Quelle ordnungsgemäß nennen, einen Link zur Creative Commons Lizenz beifügen und angeben, ob Änderungen vorgenommen wurden.

Die in diesem Kapitel enthaltenen Bilder und sonstiges Drittmaterial unterliegen ebenfalls der genannten Creative Commons Lizenz, sofern sich aus der Abbildungslegende nichts anderes ergibt. Sofern das betreffende Material nicht unter der genannten Creative Commons Lizenz steht und die betreffende Handlung nicht nach gesetzlichen Vorschriften erlaubt ist, ist für die oben aufgeführten Weiterverwendungen des Materials die Einwilligung des jeweiligen Rechteinhabers einzuholen.

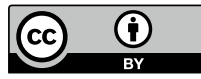

